\title{
CHCA-Modified Au Nanoparticles for Laser Desorption Ionization Mass Spectrometric Analysis of Peptides
}

\author{
Jicheng Duan, Matthew J. Linman, Chih-Yuan Chen, and Quan J. Cheng \\ Department of Chemistry, University of California-Riverside, Riverside, California, USA
}

Gold nanoparticles (AuNPs) have been studied as a potential solid-state matrix for laser desorption/ionization mass spectrometry (LDI-MS) but the efficiency in ionization remains low. In this report, AuNPs are capped by a self-assembled monolayer of cysteamine and modified with $\alpha$-cyano-4-hydroxycinnanic acid (CHCA) for effective MALDI measurements. CHCA-terminated AuNPs offer marked improvement on peptide ionization compared with citrate-capped or cysteamine-capped AuNPs. The coating also effectively suppresses formation of $\mathrm{Au}$ cluster ions and analyte fragment ions, leading to cleaner mass spectra. Addition of glycerol and citric acid to the peptide/AuNPs sample further improves the performance of these AuNPs for LDI-MS analysis. Glycerol appears to enhance the dispersion of AuNPs in sample spots, increasing the sample ionization and shot-to-shot reproducibility, while citric acid serves as an external proton donor, providing high production of protonated analyte ions and reducing fragmentation of peptides on the nanoparticle-based surface. Optimal ratios of citric acid, glycerol, and AuNPs in sample solution have been systematically studied. A more than 10-fold increase for desorption ionization of peptides can be achieved by combining $5 \%$ glycerol and $20 \mathrm{mM}$ citric acid with the CHCA-terminated AuNPs. The applicability of the CHCA-AuNPs for LDI-MS analysis of protein digests has also been demonstrated. This work shows the potential of AuNPs for SALDI-MS analysis, and the improvement with chemical functionalization, controlled dispersion, and use of an effective proton donor. (J Am Soc Mass Spectrom 2009, 20, 1530-1539) (c) 2009 Published by Elsevier Inc. on behalf of American Society for Mass Spectrometry

$\mathrm{M}$ atrix assisted laser ionization/desorption mass spectrometry (MALDI-MS) is one of the most powerful tools for analysis of large biomolecules, such as peptides, proteins, and nucleic acids [1-7]. Typical sample preparation involves mixing the analyte with organic UV-absorbing matrices. As the signal quality is highly dependent on the crystal formation, ideal conditions for good crystal quality often require excessive amounts of matrix (ca. 1000× molar excess) to achieve highly efficient ionization and desorption. Additionally, "sweet-spots" often leads to poor shot-to-shot and sample-to-sample reproducibility, thus making quantitative analysis difficult.

In recent years, surface assisted laser ionization/ desorption mass spectrometry (SALDI-MS) has been developed to offer alternative approaches to circumvent these problems. Various inorganic materials have been applied for MS analysis, including cobalt powder, graphite powder, porous silicon surface, and metal particles [8]. SALDI not only improves the reproducibility of MS analysis but also reduces the background signals of the organic matrix ions in the low-mass range

Address reprint requests to Professor Q. J. Cheng, Department of Chemistry, University of California-Riverside, Riverside, CA 92521, USA. E-mail: quan.cheng@ucr.edu
$[9,10]$. Recently, nano-materials have attracted considerable attention for SALDI-MS analysis due to their high surface areas, simple sample preparation, and flexible sample deposition conditions. Various nanomaterials, including carbon nanotubes, porous silicons, metal, and metal oxide nanoparticles have been used in SALDI-MS for analysis of biological samples [11, 12].

Gold nanoparticles (AuNPs), which display strong surface plasmon absorption properties, have been utilized in various analytical techniques, including biological optical imaging, biosensing, and surface-enhanced Raman spectroscopy [11-13]. Their potential in SALDI-MS has been recognized in recent years. AuNPs affinity probes have been applied for microextraction and concentration of peptides in aqueous solution before MS analysis [14]. $\omega$-Alkylthiol carboxylic acid functionalized AuNPs have been used for selective concentration of peptides with low isoelectric points [15]. AuNPs for SALDI-MS analysis of peptides and small protein were recently demonstrated by McLean et al. [16] on 2-10 nm particles with low ratio of matrix-to-analyte (ca. $1 \mathrm{NP} / 1 \times$ $\left.10^{7}-10^{9}\right)$. The results show AuNPs can provide the capacity for ionization of more than one analyte per laser pulse. AuNPs have also been applied for SALDIMS analysis of other samples, including aminothiols [17-19], triphosphate [19], small carbohydrates [20, 21], 
as well as oligosaccharides [18]. The use of AuNPs in SALDI-MS offers several advantages, including ease of preparation and chemical modification, high absorption coefficient, independence from irradiation wavelength, and excellent stability and biocompatibility. As certain molecules can be selectively captured on the nanoparticle surface, UV energy from the laser can be efficiently transferred for desorption and ionization of the analyte of interest. Therefore, molecules with poor ionization efficiency in traditional MALDI-MS, for instance saccharides, can be analyzed by cationization in SALDI-MS with AuNPs [18, 20, 21].

Although AuNPs have shown promise for SALDI-MS, they still suffer from some drawbacks in application [22]. First, the performance of AuNPs assisted LDI-MS appears to be sensitive to the size of the NPs. The abundance of higher-order Au clusters increases considerably as AuNP size increases [16]. Second, alkali adducted analyte ions are dominant in SALDI-MS spectra, leading to poor generation of protonated ions $[16,17,20]$. The alkali adductions complicate the identification of target analytes from complex samples. The ionization efficiency is still relatively low, and the presence of proton donor appears to be important for LDI of polar biomolecules. Recently, Castellana and Russell [22] have reported that tailoring of AuNPs surface with proton donor molecules enhances the laser desorption ionization and reduction of alkali adducted ions. It appears to be possible to improve sample ion production and UV energy-transfer via chemical modification of the NPs surface.

In this report, we examine the functionalization of AuNPs with organic acids for direct ionization of peptides. $\alpha$-Cyano-3-hydroxy-cinnamic acid (CHCA), a popular MALDI matrix, is used as functional reagent to cap the surface of AuNPs through a self-assembled monolayer (SAM) of cysteamine as the spacer. The performance of CHCA-terminated AuNPs is investigated for SALDI-MS analysis of peptides, and the results are compared with traditional organic matrix and NPs modified with citrate and cysteamine alone. To enhance the ionization efficiency, several additives, including glycerol, organic acids, and ammonium salts are tested, and the effectiveness of these additives on improving ionization with AuNPs is systematically studied. Finally, CHCA-terminated AuNPs are further tested with protein digest from cytochrome $c$ to explore the feasibility of using these NPs in protein analysis.

\section{Experimental}

\section{Chemicals}

Gold (III) chloride trihydrate, sodium citrate, $\alpha$-cyano-3hydroxy-cinnamic acid (CHCA), 1,1-carbonyl-diimidazole (CDI), cysteamine hydrochloride, formic acid, acetic acid, trifluoroacetic acid (TFA), N,N'-dimethylformamide anhydrous (DMF), cytochrome $c$, TPCK-treated trypsin, [Sar ${ }^{1}$, $\mathrm{Thr}^{8}$ ]-angiotensin II (MW = 956.1), and neurotensin (MW = 1672) were received from Sigma-Aldrich (St. Louis, MO). Citric acid, ammonium acetate, ammonium citrate, and ammonium bicarbonate were purchased from Fisher Scientific (Pittsburgh, PA).

\section{Preparation of $A u N P S$}

$\mathrm{Au}$ nanoparticles were prepared by citrate reduction of $\mathrm{HAuCl}_{4}$ using a published protocol $[19,23]$. A $200 \mathrm{~mL}$ aqueous solution containing $1.0 \mathrm{mM} \mathrm{HAuCl}_{4}$ was poured into a round-bottom flask fitted with a reflux condenser. The solution was heated to boil and vigorously stirred by a magnetic stir bar. A $20 \mathrm{~mL}$ solution of $38.8 \mathrm{mM} \mathrm{Na}_{3} \mathrm{C}_{6} \mathrm{H}_{5} \mathrm{O}_{7}$ was then added to the boiled solution quickly and allowed to react until it showed a deep red color. The size of the AuNPs was measured by use of a dynamic light scattering analyzer from Brookhaven Instruments (Holtsville, NY). UV-Vis absorbance spectra (Figure S1 in Supplement, which can be found in the electronic version of this article) shows that the maximum wavelength of the absorbance is 524 nm. These citrate-capped AuNPs were concentrated to $\sim 38 \mathrm{nM}$ by centrifugation at $12,000 \mathrm{rpm}$.

\section{Modification of $A u N P$}

The AuNP modification process is shown in Scheme 1. Cysteamine reaction solution $(20 \mathrm{mM})$ was prepared by

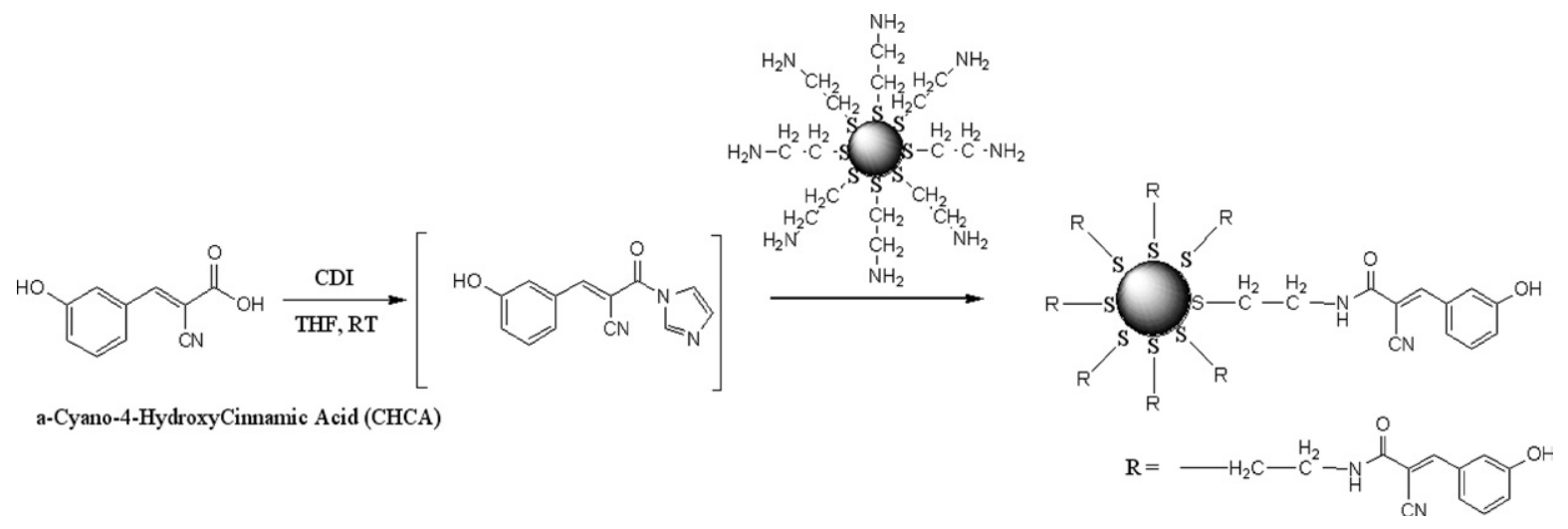

Scheme 1. Modification of cysteamine-capped AuNPs with CHCA. 
dilution of $1 \mathrm{M}$ cysteamine with $38.8 \mathrm{mM}$ trisodium citrate solution. One $\mathrm{mL}$ concentrated AuNP solution was then added into the cysteamine solution drop by drop with the assistance of supersonication. The color of the solution turned to blue when AuNPs was added. The mixture was supersonicated for another $30 \mathrm{~min}$ to complete the reaction. The excess cysteamine and citrate were removed by centrifugation-washing cycle with DMF and $50 \%$ acetonitrile $/ \mathrm{H}_{2} \mathrm{O}$ three times. For coupling with CHCA, cysteamine-capped AuNPs were dried after washing with DMF. The AuNPs were then added into $80 \mathrm{mM}$ mixture of CHCA and CDI (1:1) in $\mathrm{DMF}$ and incubated overnight at room temperature. The unreacted components were removed by a centrifugationwashing cycle with DMF and $50 \%$ acetonitrile $/ \mathrm{H}_{2} \mathrm{O}$ three times. Solutions for cysteamine-capped AuNPs and CHCA-terminated AuNPs were prepared by resuspending nanoparticles in $50 \%$ acetonitrile.

\section{Sample Preparation}

The stock solution for peptides was prepared by dissolving $\left[\mathrm{Sar}^{1}, \mathrm{Thr}^{8}\right]$-angiotensin II $(\mathrm{MW}=956.1)$ and neurotensin ( $\mathrm{MW}=1672$ ) in $50 \%$ acetonitrile to a concentration of $200 \mu \mathrm{M}$, respectively. CHCA solution $(10 \mathrm{mg} / \mathrm{mL})$ was prepared in $50 \%$ ACN water solution containing $0.1 \%$ TFA. When CHCA was used as matrix, sample solution was prepared in a 1:10 ratio of peptide solution to CHCA. When AuNPs were used as matrix, $1 \mu \mathrm{L}$ of peptide solution was mixed with $1 \mu \mathrm{L}$ AuNPs solution, followed by suspending the mixture in $50 \%$ acetonitrile at $10 \times$ dilution. Different acids, ammonium salts, and glycerol were added depending on experiment conditions.

Cytochrome $c$ solution $(1 \mathrm{mg} / \mathrm{mL})$ was prepared in $50 \mathrm{mM} \mathrm{NH}_{4} \mathrm{HCO}_{3}(\mathrm{pH} 8.0)$ and denaturized at $50{ }^{\circ} \mathrm{C}$ for $1 \mathrm{~h}$. After that, the protein was digested by TPCKtreated trypsin at $37^{\circ} \mathrm{C}$ for $24 \mathrm{~h}$ with a protein/enzyme ratio of 50:1. Formic acid was added to stop the reaction. Desalting of protein digests was carried out by using C-18 HyperSep Tips from Thermo Scientific (Waltham, MA). Desalted sample was dried under vacuum and redissolved in 50\% acetonitrile containing $10 \mathrm{mM}$ citric acid. Sample preparation of protein digests for MALDI-MS analysis is similar to that of peptides. For MALDI-MS analysis, $1 \mu \mathrm{L}$ of sample solution was deposited onto the MALDI sample plate and dried at vacuum before MS detection.

Scanning electron microscopy (SEM) images for sample spots containing AuNPs were obtained by a Philips (Hillsboro, OR) XL30 FEG scanning electron microscope system.

\section{LDI-TOF MS}

Laser desorption and matrix assisted laser desorption/ ionization mass spectra were obtained by using VoyagerDE STR MALDI-TOF mass spectrometer (Applied Biosystems, Framingham, MA) operating in positive reflec- tor mode. This mass spectrometer is equipped with a pulsed nitrogen laser operated at $337 \mathrm{~nm}$ with 3 nsduration pulses. The accelerating voltage, grid voltage, and extraction delay time were set as $20 \mathrm{kV}, 65 \%$, and $190 \mathrm{~ns}$, respectively. MS spectra were acquired as an average of 100 laser shots. For verification of CHCAmodification on NP surface, the mass spectrometer was operated in negative reflector mode. The extraction delay time was set at 110 ns.

Peptide mass mapping was carried out by comparing the mass spectra with the protein sequence database in ExPASy (Expert Protein Analysis system, http:// www.uniprot.org).

\section{Results and Discussion}

\section{Functionalization of Nanoparticles and Direct Ionization of Peptides}

The dimensions of the nanoparticles used in this study have been determined by dynamic light scattering [24], which gave an average size of $21.5 \pm 0.4 \mathrm{~nm}$ for the citrate-capped AuNPs. UV-Vis spectra show a maximum absorbance band at $524 \mathrm{~nm}$ (Figure S1 in Supplement), also indicating the formation of AuNPs with an average size of $\sim 20 \mathrm{~nm}$ [25]. As surface modification of AuNPs by thiolates is effective for manipulating the affinity property, we chose to first investigate cysteamine as the capping reagent because of its potential to enhance ionization in SALDI-MS as a proton donor.

The presence of two terminal functional groups in cysteamine, mercapto (-SH) and amino group $\left(-\mathrm{NH}_{2}\right)$, can lead to both covalent and electrostatic binding to the gold surface when substituting the citrate groups [26]. Formation of 3D crosslinking structure and aggregation of AuNPs have been observed when cysteamine and other similar bifunctional capping reagents, such as aminothiophenol and amino acids, are used in nanoparticle functionalization under acidic conditions [27, 28]. To solve this problem, a high concentration of cysteamine (i.e., $20 \mathrm{mM}$ ) was prepared in sodium citrate solution $(38.8 \mathrm{mM}, \mathrm{pH}$ 8.4) for the capping process. Under this condition, the amino groups of cysteamine are prevented from protonation, and their bonding to the gold surface is suppressed. As a result, the chemisorption of mercapto groups to AuNP surface dominates the process, building up an amino-terminated surface for AuNPs via a self-assemble mechanism.

UV-absorbing molecules, such as CHCA [29, 30], 2,5-dihydroxybenzoic acid [31, 32], and selfassembled monolayer reagents such as methyl N-(4mercaptophenyl)carbamate [33], have been immobilized on the solid support surface to assist the desorption ionization of samples in SALDI-MS. Presence of a proton donor and high extinction coefficients at laser exciting wavelengths are crucial for MALDI [34] and SALDI-MS [22, 35]. CHCA is widely used because of its high UV-absorbing property and its ability to function as an effective proton donor. It is thus chosen 
in this work as the terminal molecule on the AuNPs surface. Previously, the phenolic group has been validated as a more effective proton donor than the carboxyl group in CHCA [34]. Therefore, carboxyl group is used for linking by forming a peptide bond with the amino groups on cysteamine-capped AuNPs. A redshift in absorption spectra was observed when the two reagents were mixed (Figure S1 in Supplement). Peak broadening was also observed concurrently with color change of AuNP solution from blue to light purple, suggesting the increase in the particle size of AuNPs by modification of CHCA on the surface.

Positive verification of CHCA modification was carried out with negative mode MS, which is highly effective for identifying molecules on a AuNP surface $[19,36]$. Figure 1 shows negative mode LDI-MS spectra for different reagents capped AuNPs. Figure 1a-d illustrate results for citrate-capped AuNPs, cysteaminecapped AuNPs, CHCA-terminated AuNPs, and CHCA matrix, respectively. Au tends to exist as anion in negative mode MS detection, appearing at $m / z 197$ with high intensity in all spectra from three AuNPs surfaces, while peaks at $m / z 58,79$, and 97 appear to arise from contaminates in AuNPs solution. Peaks at $m / z 191$ and 145 were detected on the surface of citrate-capped AuNPs, corresponding to dihydrogen citrate anion and anion of hydrogen citrate with loss of carboxyl, respectively. For cysteamine-capped AuNPs, an oxidized derivate of cysteamine at $m / z 124$ was detected, providing the evidence for capping of cysteamine on the surface of AuNPs [33]. Additionally, dihydrogen citrate anions were found on the surface of cysteaminecapped AuNPs. This result suggests that although Au-S bond formation has been deemed as the most energetically favorable bond formed on gold, it might be difficult for cysteamine to completely replace all citrate molecules absorbed to the gold surface [26].

For CHCA-terminated AuNPs, peaks at $m / z$ 66, 118, and 144 were assigned for fragment ions from CHCA by comparison with MS spectrum for CHCA matrix. Peak at $\mathrm{m} / \mathrm{z} 144$ was assigned for [CHCA-COOH] ', while peak at $\mathrm{m} / \mathrm{z} 118$ assigned for [CHCA-CN-COOH$]^{-}$. The latter could also be found in CHCA matrix when the intensity of laser shot was increased. Peak at $m / z 66$ was assigned for [CHCA- $\left.\mathrm{C}_{6} \mathrm{H}_{4}(\mathrm{OH})-\mathrm{OH}\right]^{-}$, which was a unique fragment from immobilized CHCA according to the fragment mechanism. These results provide direct evidence for the successful immobilization of CHCA on the AuNP surface. Peak at $m / z 124$, which was assigned for oxidized derivate of cysteamine, appeared in Figure 1c. It suggests residues of unreacted cysteamine on the surface of CHCA-terminated AuNPs, possibly due to the steric effect of CHCA molecules on the nanoparticle surface.

The use of AuNPs for direct ionization of peptides was demonstrated with [Sar1, Thr8]-angiotensin II (M1) and neurotensin (M2). Figure 2 shows the MS spectra with citrate-capped AuNPs, cysteamine-capped AuNPs, and CHCA-terminated AuNPs. Both peptides could be found in these MALDI spectra, where peaks at $m / z$ 957, 979, 995, 1673, 1695 , and 1712 are assigned for $[\mathrm{M} 1+\mathrm{H}]^{+},[\mathrm{M} 1+\mathrm{Na}]^{+}$, $[\mathrm{M} 1+\mathrm{K}]^{+},[\mathrm{M} 2+\mathrm{H}]^{+},[\mathrm{M} 2+\mathrm{Na}]^{+}$, and $[\mathrm{M} 2+\mathrm{K}]^{+}$, respectively. When citrate-capped AuNPs were used as matrix, sodium and potassium adducted ions resulting from sodium citrate solution for stabilization of AuNPs were dominant in the spectrum. This result was similar

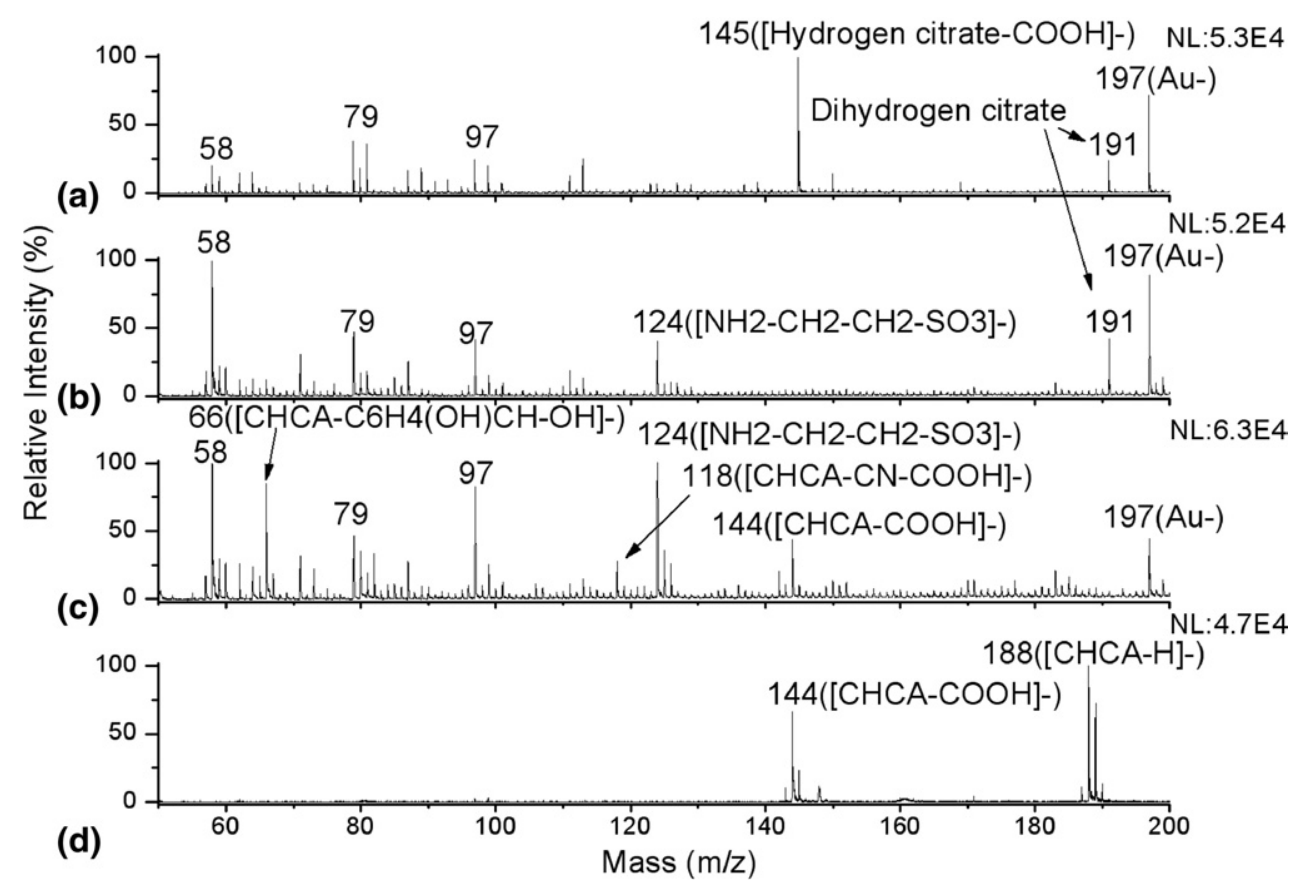

Figure 1. Negative ion mode MS spectra obtained directly with the (a) citrate-capped AuNPs, (b) cysteamine-capped AuNPs, (c) CHCA-terminated AuNPs, and (d) CHCA matrix. 


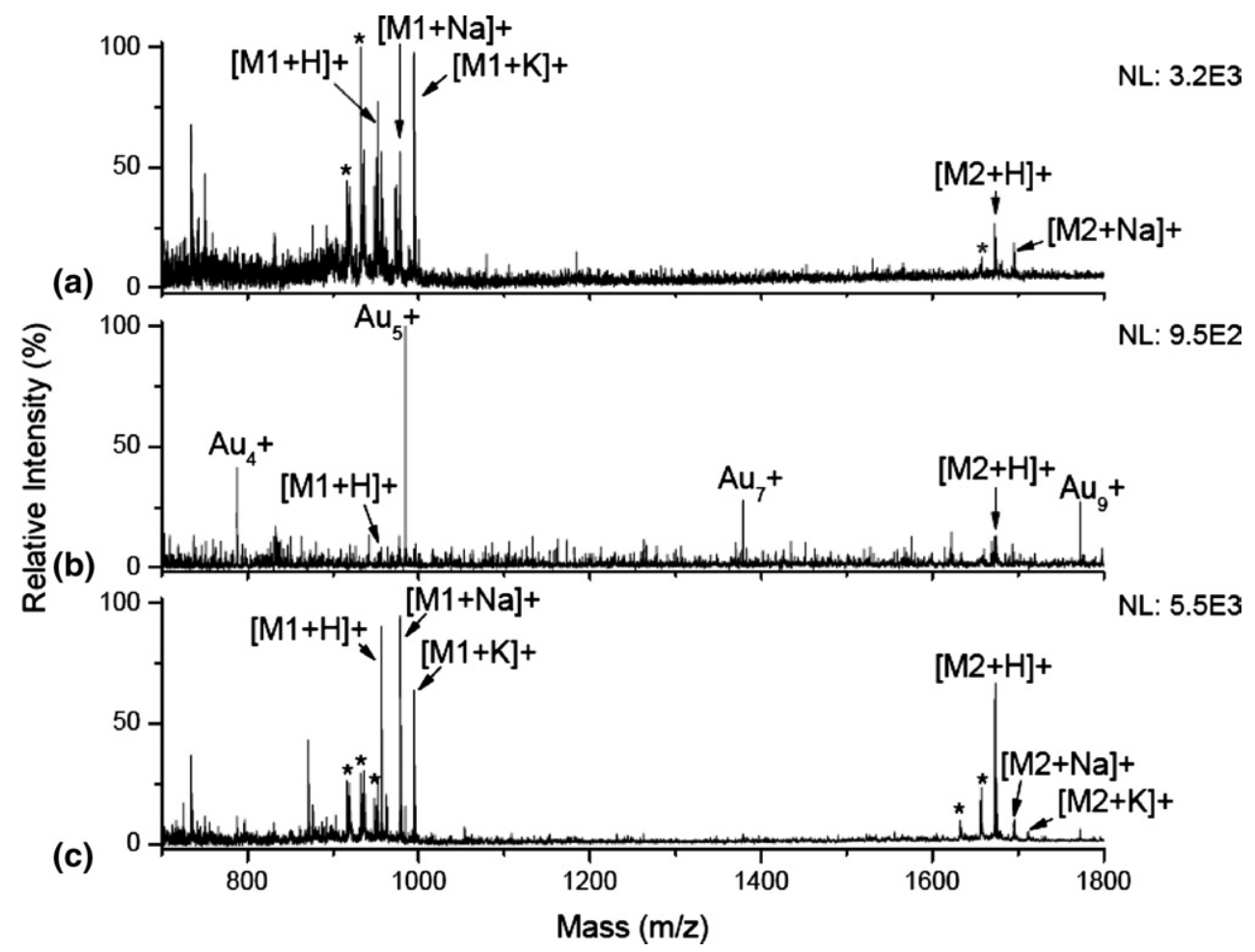

Figure 2. LDI-MS analysis of peptides with (a) citrate-capped AuNPs, (b) cysteamine-capped AuNPs, and (c) CHCA-terminated AuNPs. Sample contains 20 pmol of [Sar $\left.{ }^{1}, \mathrm{Thr}^{8}\right]$-angiotensin II $(\mathrm{MW}=956.1)$, and neurotensin $(\mathrm{MW}=1672)$ in each spot.

to those obtained on the surface of 10-nm AuNPs [16]. Additionally, serious fragmentation of peptides was observed on the nanoparticle surface, yielding several fragment ion peaks with lower $\mathrm{m} / \mathrm{z}$ value in the MS spectrum (marked with asterisk in Figure 2a). Suppression of peptide ionization was observed when citrate ions were substituted by cysteamine. Figure $2 \mathrm{~b}$ shows that two peptides yielded responses at the level of limit-of-detection with cysteamine-capped AuNPs, while abundant Au cluster ions were dominant in the spectrum. This result can be explained by the high proton affinity of the amine group in cysteamine. With a pKa value of 10.8 [37, 38], cysteamine molecules on AuNPs surface play the role of proton predators instead of proton donors [22]. Thus, because of the poor AuNPs-to-sample energy-transfer, $\mathrm{Au}$ ions themselves, not peptides, tend to be ionized when a laser pulse is applied. After capping cysteamine with CHCA, the new AuNPs exhibit improvement of desorption ionization for both peptides, yielding nearly twice higher ion responses, better signal-to-noise ratios, and less fragmentation of peptides relative to citratecapped AuNPs (Figure 2c). Additionally, protonated analyte ions tend to become dominant in MS spectra. This result clearly shows that the CHCA molecule is a highly promising terminated reagent for AuNPs to improve the ionization of the peptide samples.

It should be noted that these capped AuNPs tend to aggregate and precipitate at the edge of the sample spot resulting from inhomogeneous evaporation. This is a particularly severe problem when normal solvents com- patible with MALDI-MS analysis, such as $\mathrm{ACN} / \mathrm{H}_{2} \mathrm{O}$ and methanol $/ \mathrm{H}_{2} \mathrm{O}$, are used to build up the analyte layer on the sample plate for SALDI analysis. SEM has been used to characterize the particle aggregation, and the results are shown in Figure 3. Figure 3a-c are SEM images of sample spot prepared with $50 \%$ ACN containing $0.1 \%$ TFA. After drying, AuNPs were repelled to the edge of the spot and aggregated into larger particles, resulting in an inhomogeneous dispersion of nanoparticles. As a consequence, MS signal can be obtained only at the edge parts of the sample spot, giving poor shot-to-shot reproducibility. Additionally, the ionization efficiency declines as a result of formation of larger aggregates [16]. Therefore, it is necessary to find a new solvent system for sample preparation with AuNPs.

\section{Improving Dispersion of AuNPs on the Sample Plate}

Glycerol has been used for dispersing AuNPs without causing aggregation [39]. In addition, glycerol can serve as proton donor to assist ionization of sample in SALDI-MS [40-42]. We tested the effect of glycerol on the CHCA-terminated AuNPs. Figure 4a shows the mass spectrum of peptides using CHCA-terminated AuNPs with 5\% glycerol. Compared with the result without glycerol addition, a minor improvement in ion intensity was observed for analyte ions (less than 3 -fold) as well as fragment ions of analytes. We noticed 

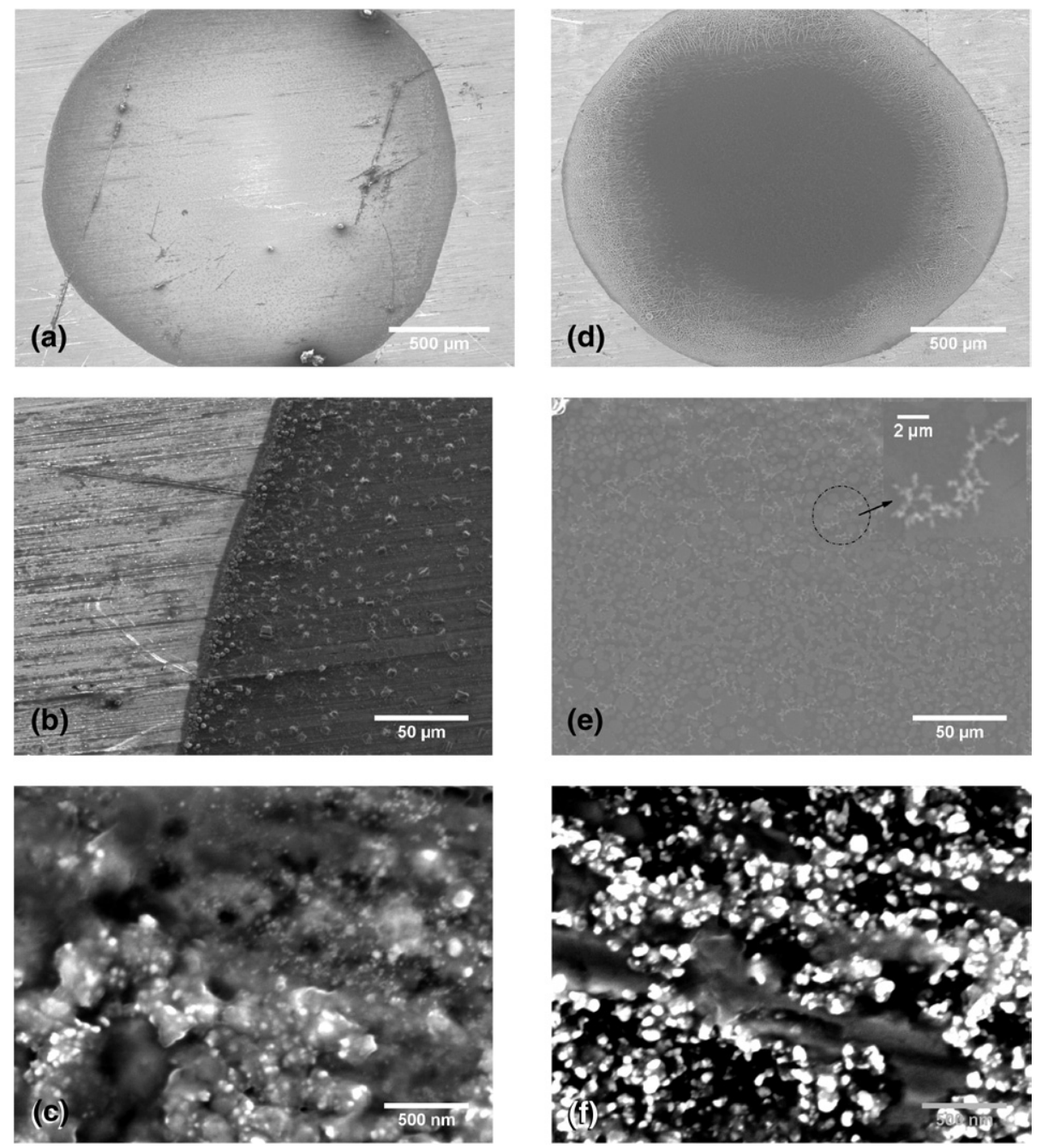

Figure 3. SEM images for sample spots prepared with CHCA-terminated AuNPs after drying under vacuum. Images (a)-(c) (left column) are for AuNPs deposited directly on the sample plate with amplification of $\times 100, \times 1000$, and $\times 90,000$, respectively; Images (d)-(f) (right column) are for AuNPs deposited with existence of $5 \%$ glycerol.

that glycerol was less effective for neurotensin, which has higher mass relative to [Sar1, Thr8]-angiotensin. Apparently, glycerol alone is not an effective proton donor for metal nanoparticle-based approach. A similar result has been reported previously in the study of silver nanoparticle matrix for SALDI-MS [43]. However, the dispersion of AuNPs in the sample spot was substantially improved. Figure $3 \mathrm{~d}-\mathrm{f}$ show the sample spot prepared with addition of glycerol by SEM. AuNPs are dispersed homogeneously over the sample spot without serious aggregation. The particle size in sample spot is in tens of nanometers (Figure 3f) compared with several hundred nanometers for those without glycerol (Figure 3c). CHCA-terminated AuNPs exhibit "grapelike" aggregation over the sample spot with the presence of glycerol (Figure 3e), while the extent of aggregation of AuNPs has been considerably improved compared with those in solution situation [26, 28]. This result also provides an explanation for the observation of particle size increasing in UV-Vis absorption spectra.
Importantly, stable and consistent sample signal can be achieved over the whole sample spot with glycerol addition. Clearly, glycerol plays a great role in improving dispersion and, thus, reproducibility, but not necessarily improving ionization in SALDI-MS analysis.

Additional tests were carried out using citric acid as an effective proton source for SALDI-matrixes [35, 44, 45]. Figure $4 \mathrm{~b}$ shows the improvement of signal-tonoise ratio for protonated peptide ions when citric acid was added. Alkaline metal adducted ions and fragment ions were eliminated or reduced with citric acid in sample spot. More importantly, productions of analyte fragment ions were totally suppressed. However, inhomogeneity of sample spot and relative poor detection intensity resulted with citric acid alone. Based on these results, dispersion of AuNPs in glycerol with addition of citric acid would be a rational choice for further investigation. Figure 4c shows the MS spectrum obtained with CHCA-terminated AuNPs in the presence of 5\% glycerol and $20 \mathrm{mM}$ citric acid. The intensities of both peptides 


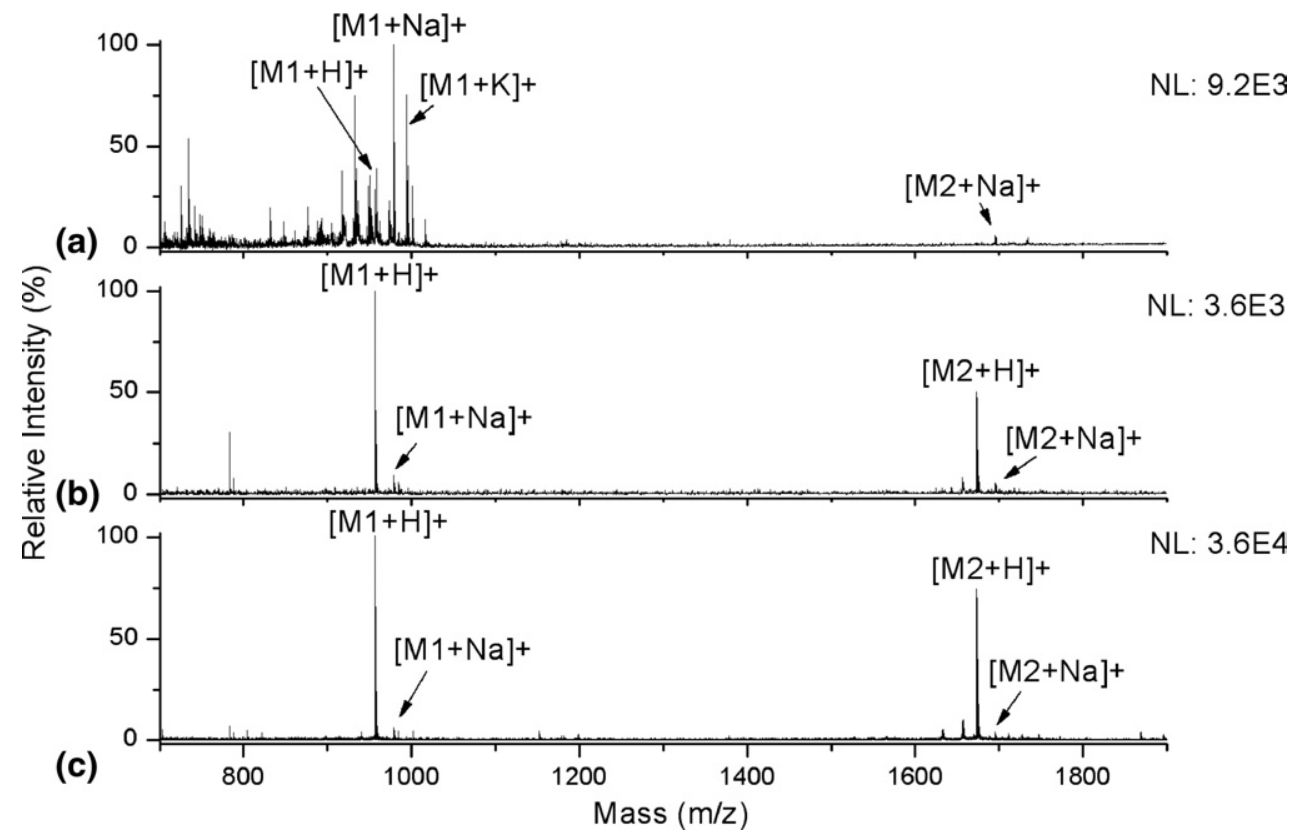

Figure 4. LDI-MS spectra for peptides on the surface of CHCA-terminated AuNPs, (a) with addition of $5 \%$ glycerol in sample solution; (b) with addition of $20 \mathrm{mM}$ citric acid in sample solution; (c) with addition of $20 \mathrm{mM}$ citric acid and $5 \%$ glycerol in sample solution.

were improved nearly 10-fold compared with that with 20 $\mathrm{mM}$ citric acid alone, and high quality MS spectrum can be obtained over the whole homogenous sample spot without adverse fragmentation of peptides. Detailed analysis and comparison of ion intensity under various conditions are presented in Figure S2 in Supplement. Additionally, we found that neither citric acid nor glycerol alone can give satisfactory results without the existence of AuNPs due to the relatively low absorption of the two materials at $337 \mathrm{~nm}$. It means AuNPs are important for absorbing UV energy. From this work, we can draw some conclusions for the AuNPs-based ionization process investigated here. AuNP appears to function as an antenna for receiving UV-energy from the laser source [16], which will be transferred to molecules for desorption/ ionization. CHCA on the AuNP surface plays a bridging role that relays the energy. It also functions as proton donor as well as UV-absorbing species. Glycerol increases the viscosity of the sample solution and suppresses the inhomogeneous distribution of AuNPs caused by particle aggregation after solvent evaporation. More importantly, because of its low thermal conductivity, this liquid matrix more likely serves as a "reservoir" to lock the energy in the surrounding area of the UV-activated AuNPs [46, 47], leading to more efficient UV-energy-transfer. The addition of citric acid provides an external proton source for protonation of peptides, resulting in a high, abundantly protonated-ion-dominant MS spectrum.

\section{Effect of External Proton Donors on LDI}

To further investigate the effect of external proton source on peptide ionization with AuNPs, various acids and ammonium salts, including TFA, acetic acid, ammonium acetate, and ammonium citrate, have been added to the mixtures of sample/AuNPs before MS analysis. Table S1 in Supplement shows the signal intensity for MS detection of peptides with different organic acids and salts. In addition, results obtained with these external proton donors in the presence of $5 \%$ glycerol were also shown for comparison. For samples without glycerol, alkali adducted analyte ions were dominated in MS spectra with fragment ions. Addition of citric acid and ammonium citrate can increase ionization efficiency and reduce fragmentation of peptides, resulting in better results over other additives, as shown with higher MS intensity of peptides. Between them, ammonium citrate appeared to offer more promising results compared with citric acid, where ionization of neurotensin at higher mass weight was enhanced. However, inhomogeneous distribution of nanoparticles remained a problem when ammonium salts were used, which resulted in the similar morphology of sample spot showed in Figure 3a. Addition of glycerol offers benefit for better dispersion of AuNPs as well as preserving volatile materials. We observed that for highly volatile compounds, such as TFA, acetic acid, and ammonium acetate, the intensities for MS detection of peptides were considerably improved when glycerol is present compared to those without glycerol. In the case of $0.1 \% \mathrm{TFA}$, for instance, the abundance for sodium adducted ions of angiotensin II and neurotensin were improved by 27 and 9 times with the assistance of glycerol, respectively. However, no significant improvement was observed for the MS intensity of peptides in ammonium citrate matrix system after adding 
glycerol. Since ammonium citrate is stable under vacuum, the composition in these matrix systems is expected to remain constant except for the loss of solvent under vacuum. In contrast, highly volatile acids and salts tend to evaporate along with the solvent, leading to a loss of external proton donors for ionization and, thereafter, reduction of MS response. Citric acid is also nonvolatile under vacuum, but its acidic property would benefit proton transfer from matrix to sample with the assistance of glycerol. As a result, ion intensity for peptides in citric acid/glycerol was noticeably improved compared with the ion intensity with ammonium citrate/glycerol. Although the improvement of ion intensity for sodiumated ions in citric acid/glycerol was just 4.8 times for angiotensin II and 3.2 times for neurotensin, which were not so large relatively, protonated ions tended to become dominant in spectra and showed highest ion intensities compared with those with other additives. This result suggests that the combination of citric acid and glycerol would be the best choice for peptide analysis.

The performance of other functionalized AuNPs with citric acid/glycerol additives was investigated for comparison purposes. In general, glycerol and citric acid allow consistent and abundant MS signals to be acquired with AuNPs, which dispersed homogeneously over the sample spot, without the requirement for sweet-spot searching. When citrate-capped AuNPs were used, sodium adducted analyte ions appeared as the most abundant peaks in the MS spectrum, due to sodium citrate molecules binding on the AuNP surface. In contrast, protonated peptide ions were dominant when using cysteamine-capped and CHCA-terminated AuNPs (Figure S3 in Supplement). Ionization efficiency improved noticeably with modification of AuNPs, resulting in relative higher response in MS detection. Additionally, as expected, both citrate-capped and cysteamine-capped AuNPs tended to produce more Au cluster ions compared with CHCA-terminated particles when high laser intensity was exerted (Figure S3 in Supplement). This result suggests that energy-transfer from CHCA-terminated AuNPs for analyte ionization appears to be the most efficient among all AuNPs studied.

\section{Optimization of Conditions for Effective Ionization}

The effect of citric acid on SALDI-MS analysis of peptides using CHCA-terminated AuNPs has been systematically optimized. Samples prepared in different concentrations of citric acid were subjected to MS analysis. It was found that the MS response for peptides was improved with the increasing amount of citric acid in sample matrix (Figure S4 in Supplement). The ion intensities of protonated ions were increased about 6.5 and 20 times for angiotensin II and neurotensin, respectively, when concentration of citric acid in sample was increased from $1 \mathrm{mM}$ to $20 \mathrm{mM}$. However, when more than $20 \mathrm{mM}$ citric acid was attempted, citric acid tended to crystallize after solvent evaporation, resulting in a "hard" sample spot under microscopic observation. The cocrystallization of citric acid with AuNPs alters the dispersion of nanoparticles and reduces the efficiency of energy-transfer to sample molecules, leading to the reduction of MS response. For instance, ion intensities of protonated ions decreased by about $20 \%$ and $22 \%$ for angiotensin II and neurotensin with $50 \mathrm{mM}$ citric acid, respectively, relative to those with $20 \mathrm{mM}$ citric acid.

Glycerol is important for sample desorption/ionization when particle-based matrixes were used [40], and its role has been verified in our work. The optimal percentage of glycerol was investigated, and we found that the MS response for protonated ions was increased by 3.5 and 3.7 times for angiotensin II and neurotensin, respectively, with increase in glycerol from $0.5 \%$ to $5 \%$ in the mixture of sample/AuNPs (Figure S5 in Supplement). However, higher content of glycerol in the sample would cause serious vacuum problem in MS spectrometer. So, $5 \%$ glycerol has been used for sample preparation throughout this work.

The amount of nanoparticles in the solution for optimal performance was also investigated with $\mathrm{CHCA}$-terminated AuNPs. As shown in Table 2 in Supplement, the AuNP concentration in matrix plays a positive role for enhancing the MS response of peptides. When $3.8 \mathrm{fmol}$ of AuNPs is used for sample preparation, high MS response that is comparable to that obtained by using 50 mM CHCA ( $~ 50$ nmol in sample spot) as matrix can be achieved. Therefore, the molar matrix-to-analyte ratio for CHCA-AuNPs-base MALDI is about six orders of magnitude less than traditional CHCA organic matrix. This result illustrates the remarkably high efficiency of CHCA-terminated AuNPs for desorption ionization of peptides. Additionally, there seems to be a morphological factor here that works to improve peptide ionization through the assembly of AuNPs in sample spots. We found that in the SEM image of the sample spot, AuNPs tend to assemble into multiple layer structures after drying, when high amount of AuNPs was used (Figure 3f). It may account for the enhancement of MS detection when increasing the content of AuNPs in samples. However, it should be noted that higher content of AuNPs increased the possibility of aggregation of nanoparticles in sample spot. Therefore, the concentration of CHCA-terminated AuNPs was suggested to be limited below $24.5 \mathrm{nM}$ in sample solution.

\section{Analysis of Protein Digests with AuNPs}

To validate the applicability of CHCA-terminated AuNPs to the research of proteome, the tryptic digest of cytochrome $c$ was analyzed. The mass spectrum recorded from the digest/AuNPs samples is shown in Figure 5a. A MALDI mass spectrum using CHCA matrix is shown in Figure $5 b$ for comparison. Seven peptides have been identified in both MS spectra, while the peptides from AuNPs matrix are mainly detected as their alkali adducted species, yielding amino acid sequence coverage of $60 \%$. The abundance of alkali ad- 


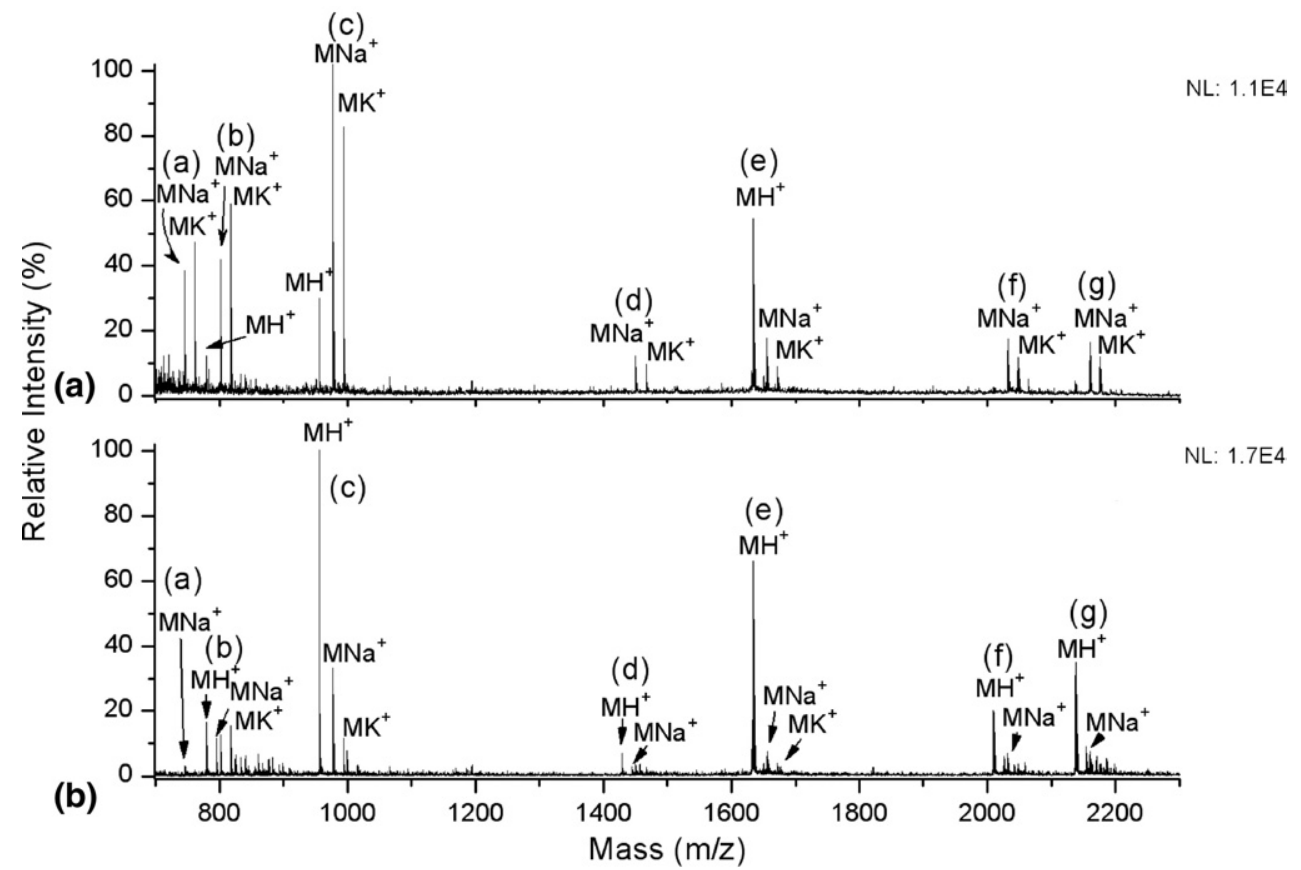

Figure 5. Mass spectra of tryptic digest from cytochrome $c$ by using CHCA-terminated AuNPs (a) and CHCA as matrix (b). Peptide (a): EDLIAY, 93-98, $\mathrm{m} / \mathrm{z}$ 723.4; peptide (b): MIFAGIK, 81-87, $\mathrm{m} / \mathrm{z}$ 779.4; peptide (c): TGPNLHGLF, 29-37, $\mathrm{m} / \mathrm{z}$ 955.5; peptide (d): GITWGEETLMEY, 57-68, m/z 1428.6; peptide (e): IFVQKCAQCHTVEK, 10-23, m/z 1633.8; peptide (f): GITWGEETLMEYLENPK, 57-73, m/z 2010.0; peptide (g): GITWGEETLMEYLENPKK, 57-74, $\mathrm{m} / \mathrm{z}$ 2138.0. Sample conditions: 80 pmol for CHCA matrix; 400 pmol for CHCA-terminated AuNPs.

ducted ions might arise from salt used in sample preparation. Among these identified peptides, three chymotryptic peptides were found and denoted as a, c, and $d$ in Figure 5. These nonspecific cleavage peptides may be generated by long time incubation/digestion. The ionization efficiency for CHCA-terminated AuNPs appears to be still relatively poor compared with that for CHCA, since more digest ( $\sim 5 \times$ excess) has to be employed for AuNPs to obtain the similar result as organic matrix. It may suggest that the sample complexity affects dispersion and, therefore, efficiency of AuNPs in the sample spot. Our current efforts are focused on looking for a more robust solvent system for improving the performance of AuNPs in analysis of complex samples. Nevertheless, the applicability of CHCAfunctionalized AuNPs in proteome MS analysis is clearly demonstrated.

\section{Conclusions}

In this work, we demonstrate the use of functional $\mathrm{Au}$ nanoparticles for LDI mass spectrometric analysis of peptides. CHCA-terminated AuNPs were prepared by immobilization of CHCA molecules to the surface of AuNPs through cysteamine spacer. The desorption/ ionization efficiency for peptides on these AuNPs is improved compared with those without CHCA modification. Another benefit of the surface modification is the suppression of the generation of Au cluster ions in MS analysis. Glycerol was found to benefit the disper- sion for AuNPs in sample spots, preventing nanoparticles from aggregation during solvent evaporation, and providing better shot-to-shot reproducibility, while citric acid, as an external proton donor, increased the production of protonated analyte ions and improved the quality of background noise. The best results have been achieved by combination of CHCA-terminated AuNPs with optimal amounts of glycerol and citric acid. This work shows the bright potential of AuNPs for SALDI-MS analysis, specifically when coupled with improved chemical functionalization, controlled dispersion, and the use of an effective proton donor.

\section{Acknowledgments}

The authors thank Dr. Richard Kondrat, Ron New, and Lei Xiong for help with the mass spectrometric measurements. The authors acknowledge financial support from NSF grant CHE-0719224.

\section{Appendix A Supplementary Material}

Supplementary material associated with this article may be found in the online version at doi:10.1016/j. jasms.2009.04.009.

\section{References}

1. Fenselau, C. MALDI MS and Strategies for Protein Analysis. Anal. Chem. 1997, 69(21), A661-A665. 
2. Jungblut, P.; Thiede, B. Protein Identification from 2-DE Gels by MALDI Mass Spectrometry. Mass Spectrom. Rev. 1997, 16(3), 145-162.

3. Nordhoff, E.; Kirpekar, F.; Roepstorff, P. Mass Spectrometry of Nucleic Acids. Mass Spectrom. Rev. 1996, 15(2), 67-138.

4. Gevaert, K.; Vandekerckhove, J. Protein Identification Methods in Proteomics. Electrophoresis 2000, 21(6), 1145-1154

5. Lahm, H. W.; Langen, H. Mass Spectrometry: A Tool for the Identification of Proteins Separated by Gels. Electrophoresis 2000, 21(11), 21052114.

6. Gross, J.; Strupat, K. Matrix-Assisted Laser Desorption/Ionization Mass Spectrometry Applied to Biological Macromolecules. Trac-Trends Anal. Chem. 1998, 17(8/9), 470-484.

7. Kussmann, M.; Nordhoff, E.; RahbekNielsen, H.; Haebel, S.; RosselLarsen, M.; Jakobsen, L.; Gobom, J.; Mirgorodskaya, E.; KrollKristensen, A.; Palm, L.; Roepstorff, P. Matrix-Assisted Laser Desorption/Ionization Mass Spectrometry Sample Preparation Techniques Designed for Various Peptide and Protein Analytes. J. Mass Spectrom. 1997, 32(6), 593-601.

8. Peterson, D. S. Matrix-Free Methods for Laser Desorption/Ionization Mass Spectrometry. Mass Spectrom. Rev. 2007, 26(1), 19-34.

9. Kawasaki, H.; Yonezawa, T.; Watanabe, T.; Arakawa, R. Platinum Nanoflowers for Surface-Assisted Laser Desorption/Ionization Mass Spectrometry of Biomolecules. J. Phys. Chem. C 2007, 111(44), 1627816283.

10. Merchant, M.; Weinberger, S. R. Recent Advancements in SurfaceEnhanced Laser Desorption/Ionization-Time of Flight-Mass Spectrometry. Electrophoresis 2000, 21(6), 1164-1177.

11. Anker, J. N.; Hall, W. P.; Lyandres, O.; Shah, N. C.; Zhao, J.; Van Duyne, R. P. Biosensing with Plasmonic Nanosensors. Nat. Mater. 2008, 7(6), $442-453$

12. Willets, K. A.; Van Duyne, R. P. Localized Surface Plasmon Resonance Spectroscopy and Sensing. Annu. Rev. Phys. Chem. 2007, 58, 267-297.

13. Zhao, J.; Zhang, X. Y.; Yonzon, C. R.; Haes, A. J.; Van Duyne, R. P. Localized Surface Plasmon Resonance Biosensors. Nanomedicine 2006, 1(2), 219-228.

14. Sudhir, P. R.; Wu, H. F.; Zhou, Z. C. Identification of Peptides Using Gold Nanoparticle-Assisted Single-Drop Microextraction Coupled with Ap-MALDI Mass Spectrometry. Anal. Chem. 2005, 77(22), 7380-7385.

15. Vanderpuije, B. N. Y.; Han, G.; Rotello, V. M.; Vachet, R. W. Mixed Monolayer-Protected Gold Nanoclusters as Selective Peptide Extraction Agents for MALDI-MS Analysis. Anal. Chem. 2006, 78(15), 5491-5496.

16. McLean, J. A.; Stumpo, K. A.; Russell, D. H. Size-Selected (2-10 Nm) Gold Nanoparticles for Matrix Assisted Laser Desorption Ionization of Peptides. J. Am. Chem. Soc. 2005, 127(15), 5304-5305.

17. Huang, Y. F.; Chang, H. T. Nile Red-Adsorbed Gold Nanoparticle Matrixes for Determining Aminothiols through Surface-Assisted Laser Desorption/Ionization Mass Spectrometry. Anal. Chem. 2006, 78(5), 1485-1493.

18. Wu, H. P.; Su, C. L.; Chang, H. C.; Tseng, W. L. Sample-First Preparation: A Method for Surface-Assisted Laser Desorption/Ionization Timeof-Flight Mass Spectrometry Analysis of Cyclic Oligosaccharides. Anal. Chem. 2007, 79(16), 6215-6221.

19. Huang, Y. F.; Chang, H. T. Analysis of Adenosine Triphosphate and Glutathione through Gold Nanoparticles Assisted Laser Desorption/ Ionization Mass Spectrometry. Anal. Chem. 2007, 79(13), 4852-4859.

20. Su, C. L.; Tseng, W. L. Gold Nanoparticles as Assisted Matrix for Determining Neutral Small Carbohydrates through Laser Desorption/ Ionization Time-of-Flight Mass Spectrometry. Anal. Chem. 2007, 79(4), 1626-1633.

21. Nagahori, N.; Nishimura, S. I. Direct and Efficient Monitoring of Glycosyltransferase Reactions on Gold Colloidal Nanoparticles by Using Mass Spectrometry. Chem. Eur. J. 2006, 12(25), 6478-6485.

22. Castellana, E. T.; Russell, D. H. Tailoring Nanoparticle Surface Chemistry to Enhance Laser Desorption Ionization of Peptides and Proteins. Nano Lett. 2007, 7 3023-3025.

23. Frens, G. Controlled Nucleation for Regulation of Particle-Size in Monodisperse Gold Suspensions. Nat. Phys. Sci. 1973, 241(105), 20-22.

24. Bogatyrev, V. A.; Dykman, L. A.; Khlebtsov, B. N.; Khlebtsov, N. G. Measurement of Mean Size and Evaluation of Polydispersity of Gold Nanoparticles from Spectra of Optical Absorption and Scattering. Opt. Spectrosc. 2004, 96(1), 128-135.
25. Brown, K. R.; Walter, D. G.; Natan, M. J. Seeding of Colloidal Au Nanoparticle Solutions. 2. Improved Control of Particle Size and Shape. Chem. Mat. 2000, 12(2), 306-313.

26. Basu, S.; Pande, S.; Jana, S.; Bolisetty, S.; Pal, T. Controlled Interparticle Spacing for Surface-Modified Gold Nanoparticle Aggregates. Langmuir 2008, 24(10), 5562-5568

27. Mohammad, A. M.; Abdelrahman, A. I.; El-Deab, M. S.; Okajima, T. Ohsaka, T. On the Aggregation Phenomena of Au Nanoparticles: Effect of Substrate Roughness on the Particle Size. Colloid Surf., Physicochem. Eng. Asp. 2008, 318(1/3), 78-83.

28. Zhong, Z. Y.; Patskovskyy, S.; Bouvrette, P.; Luong, J. H. T.; Gedanken, A. The Surface Chemistry of Au Colloids and Their Interactions with Functional Amino Acids. J. Phys. Chem. B 2004, 108(13),4046-4052.

29. Hutchens, T. W.; Yip, T. T. New Desorption Strategies for the MassSpectrometric Analysis of Macromolecules. Rapid Commun. Mass Spectrom. 1993, 7(7), 576-580.

30. Kitagawa, N. T. Synthesis of a Polyelectrolyte and Its Applications in Laser Desorption/Ionization. Anal. Chem. 2006, 78(2), 459-469.

31. Ho, K. C.; Lin, Y. S.; Chen, Y. C. Laser Desorption/Ionization Mass Spectrometry on Sol-Gel-Derived Dihydroxybenzoic Acid Isomeric Films. Rapid Commun. Mass Spectrom. 2003, 17(23), 2683-2687.

32. Lin, Y. S.; Chen, Y. C. Laser Desorption/Ionization Time-of-Flight Mass Spectrometry on Sol-Gel-Derived 2,5-Dihydroxybenzoic Acid Film. Anal. Chem. 2002, 74(22), 5793-5798.

33. Mouradian, S.; Nelson, C. M.; Smith, L. M. A Self-Assembled Matrix Monolayer for UV-MALDI Mass Spectrometry. J. Am. Chem. Soc. 1996, 118(36), 8639-8645.

34. Martic, S.; Brennan, J. D.; Brook, M. A.; Ackloo, S.; Nagy, N. Towards the Development of a Covalently Tethered MALDI System-a Study of Allyl-Modified MALDI Matrixes. Can. J. Chem. Rev. Can. Chim. 2007, 85(1), 66-76.

35. Chiu, Y. C.; Chen, Y. C. Carboxylate-Functionalized Iron Oxide Nanoparticles in Surface-Assisted Laser Desorption/Ionization Mass Spectrometry for the Analysis of Small Biomolecules. Anal. Lett. 2008, 41(2) 260-267.

36. Schaaff, T. G. Laser Desorption and Matrix-Assisted Laser Desorption/ Ionization Mass Spectrometry of $29-\mathrm{Kda} \mathrm{Au}: \mathrm{Sr}$ Cluster Compounds. Anal. Chem. 2004, 76(21), 6187-6196.

37. Katz, E.; LionDagan, M.; Willner, I. Ph-Switched Electrochemistry of Pyrroloquinoline Quinone at Au Electrodes Modified by Functionalized Monolayers. J. Electroanal. Chem. 1996, 408(1/2), 107-112.

38. Wirde, M.; Gelius, U.; Nyholm, L. Self-Assembled Monolayers of Cystamine and Cysteamine on Gold Studied by XPS and Voltammetry. Langmuir 1999, 15(19), 6370-6378.

39. Chen, H. J.; Kou, X. S.; Yang, Z.; Ni, W. H.; Wang, J. F. Shape- and Size-Dependent Refractive Index Sensitivity of Gold Nanoparticles. Langmuir 2008, 24(10), 5233-5237.

40. Schurenberg, M.; Dreisewerd, K.; Hillenkamp, F. Laser Desorption/ Ionization Mass Spectrometry of Peptides and Proteins with Particle Suspension Matrixes. Anal. Chem. 1999, 71(1), 221-229.

41. Tanaka, K.; Waki, H.; Ido, Y.; Akita, S.; Yoshida, Y.; Yoshida, T. Protein and Polymer Analyses up to $m / 100,000$ by Laser Ionization Time-ofFlight Mass Spectrometry. Rapid Commun. Mass Spectrom. 1988, 2(8), 151-153.

42. Chen, C. T.; Chen, Y. C. Molecularly Imprinted Tio2-Matrix-Assisted Laser Desorption/Ionization Mass Spectrometry for Selectively Detecting $\alpha$-Cyclodextrin. Anal. Chem. 2004, 76(5), 1453-1457.

43. Hua, L.; Chen, J. R.; Ge, L.; Tan, S. N. Silver Nanoparticles as Matrix for Laser Desorption/Ionization Mass Spectrometry of Peptides. J. Nanopart. Res. 2007, 9(6), 1133-1138.

44. Lo, C. Y.; Lin, J. Y.; Chen, W. Y.; Chen, C. T.; Chen, Y. C. SurfaceAssisted Laser Desorption/Ionization Mass Spectrometry on Titania Nanotube Arrays. J. Am. Soc. Mass Spectrom. 2008, 19(7), 1014-1020.

45. Chen, C. T.; Chen, Y. C. Desorption/Ionization Mass Spectrometry on Nanocrystalline Titania Sol-Gel-Deposited Films. Rapid Commun. Mass Spectrom. 2004, 18(17), 1956-1964.

46. Zenobi, R.; Knochenmuss, R. Ion Formation in MALDI Mass Spectrometry. Mass Spectrom. Rev. 1998, 17(5), 337-366.

47. Knochenmuss, R. Ion Formation Mechanisms in UV-MALDI. Analyst 2006, 131(9), 966-986. 